\title{
GUERRA Y PAZ: ANÁLISIS ACERCA DE KANT Y CLAUSEWITZ ${ }^{1}$
}

Carlos García Gil

Universidad Pedagógica y Tecnológica de Colombia

"El sonido que permanentemente reverbera a través
de la historia es el de los tambores de la guerra"
Koestler.
"La guerra es la salida cobarde a los problemas de
la paz"
T. Mann

\section{Resumen}

El presente artículo pretende indicar las posturas encontradas, entre Kant y Clausewitz, respecto a la cuestión de la guerra y la paz, a partir de dos textos centrales: La Paz Perpetua, del primero y De la Guerra, del segundo. Con tal finalidad se parte del planteamiento de los puntos en común entre estos autores, para desembocar, finalmente, en le esclarecimiento de los puntos en contra respecto a la guerra y, principalmente, en relación con las posturas respecto a la paz, que permiten afirmar un antagonismo prácticamente absoluto.

Palabras clave: guerra, paz, política, Estado, derecho.

\section{Abstract}

\section{War and peace : an analysis of kant and clausewitz}

This article aims at contrasting the standpoints of Kant and Clausewitz, regarding the matter of war and peace, based on two central texts: Perpetual peace, by the first author and $O n$ war by the second one. In order to achieve this, we start from the common points between these authors, to arrive, in the end, at the contrary points regarding war, and mostly, regarding their ideas about peace, which are practically in complete antagonism.

Key Words: War, peace, politics, State, law.

Imperecedera es la voluntad de lucha, ya sea ésta individual o colectiva. La permanencia de la inclinación combativa parece innegable, como la intención, fruto del temor a la catástrofe, de dejar de luchar, o al menos de suspender, por algún tiempo, la incansable inclinación de luchar. De ese modo, guerra y paz se entrelazan como tejidos de una

\footnotetext{
${ }^{1}$ Texto que forma parte del Trabajo de Grado como proyecto de investigación: "Diferencias y convergencias entre Kant y Clausewitz frente al problema de la guerra y la paz”. UPCT, 2008.
} 
realidad por esencia contradictoria, como hilos que moldean la historia plenamente. De hecho, también parece de conocimiento común según el cual la principal fuerza motriz de la guerra es la búsqueda de la paz, que convierte ésta en algo menos que un pretexto, a través del cual se justifican las demás causas de la guerra, específicamente, aquellas acciones que preceden a la confrontación como tal, como a las fases trágicas de todo conflicto. Pero la constante fatalidad humana que se expresa en la guerra hace que su contraparte se reduzca a realidad nominal, a mera aspiración, sobre todo cuando se le concibe como paz verdadera, es decir, como el conjunto de condiciones que pueden integrar a toda la especie humana, bajo esquemas que garanticen el respeto al derecho a existir, inherente a todos los pueblos. A pesar de ello, la guerra no deja ni ha dejado de ser el término fuerte, el término con un correlato en la historia, y paz un término subsidiario, ésto es, dependiente del término mayor que lo determina como consecuencia o efecto del uso de la fuerza, como resultante necesaria e ideal, también necesario, al proceso político de los pueblos.

De acuerdo con lo anterior, el objetivo del presente ensayo es la presentación sucinta del posible debate de los planteamientos de Kant y Clausewitz respecto a la guerra y la paz, a partir de dos textos, La Paz Perpetua y De la Guerra, respectivamente, que ilustran las posturas encontradas del filósofo y el pensador militar prusiano, frente a la probabilidad (teórica, o de fe racional), de una paz duradera que incluya a toda la especie humana, o la confirmación de la prevalencia de la guerra, en tanto constituye un elemento constitutivo de la naturaleza humana. No obstante, se partirá, en primer lugar, del reconocimiento de los puntos en común de dichas visiones, para desembocar, en la determinación de los puntos antagónicos, que se corresponden con el intento de señalar la complejidad de la reflexión filosófica que se puede entablar respecto a la guerra y la paz.

\section{Convergencias de contenido}

a) Convergencias generales.

Los puntos en común, las convergencias de mayor generalidad entre Kant y Clausewitz, respecto a las ideas sobre guerra y paz, son los siguientes:

-El reconocimiento común de la guerra como una constante de las interacciones entre los pueblos. La guerra como un factor inherente a la existencia política ${ }^{2}$.

-Respecto a las características de los tratados de paz, hay semejanza por cuanto constituye una pausa o conclusión no definitiva, una transición por las desventajas que expresa, por el ocultamiento natural de los tratados; y como un paso hacia otras confrontaciones. Cabe

\footnotetext{
${ }^{2}$ Hay que aclarar que mientras que para Kant la guerra es un estado previo al ingreso de la legalidad de los pueblos, la característica esencial de la guerra como elemento central del estado de naturaleza, en contraste, para Clausewitz, no es posible el estado de legalidad o de paz internacional por medio de una confederación, ni de otro tipo de organización, precisamente porque la guerra es un elemento connatural que hace parte de la esencia, si se quiere, invariable de la existencia social.
} 
aclarar: para Kant, no obstante, todos los tratados no son más que armisticios. Para Clausewitz hay una diferencia, pues el armisticio es una pausa de mayor brevedad y fragilidad respecto a un tratado; es la pausa en medio de una guerra; el tratado es la conclusión provisionalmente definitiva de una guerra en particular.

-El planteamiento según el cual la guerra es un medio y la paz un fin. Para Kant la guerra es un medio o recurso de la Naturaleza a través del cual se crean mayores espacios de legalidad, que permitan a futuro el surgimiento de la Confederación de Estados, la instauración de la paz internacional. Para Clausewitz, la guerra también es un instrumento, un recurso, pero de la política, basado en el uso de la fuerza con el fin primario de concretar los planes o intereses políticos de una nación o de un gobierno; es el medio por el que los Estados se afirman a sí mismos, buscando su preservación. Por su parte, la paz es la finalidad última de la planeación estratégica, del plan general de una guerra. Es también la finalidad última de la labor ofensiva, e indirectamente de la función defensiva. Dado que no se puede combatir por siempre, que la suspensión de la hostilidad es una regla común, la finalidad de la guerra es la paz que se otorga a favor del que obtiene las mayores ventajas, y que otorga los mayores despropósitos al que le han sido otorgados los mayores descalabros, al que resultó colmado de desventajas y debilidades, acompañadas de una mala fortuna. La paz, en ambos, es un territorio al que se llega después de las guerras.

-La idea según la cual la guerra hace parte de la naturaleza humana, y la paz como necesidad o requerimiento por el que se busca una salida a los efectos dolorosos y a veces inútiles de la guerra. La guerra es propiamente humana por cuanto corresponde, según Kant, a la maldad natural del hombre que los conduce a promover la paz como un bien prescrito por la razón. En forma análoga, la guerra para Clausewitz es estrictamente una acción humana; corresponde en esencia con la naturaleza humana; y el rasgo común de esa naturaleza es la debilidad, es decir, la tendencia contradictoria de crear cosas a las que, a la vez se les teme. Estando inscrita en la cadena de las debilidades humanas, la guerra trae consigo, como principal requerimiento, la paz, la cual, al ser producto de la guerra, es probable, no definitiva e indeterminada como la guerra. De ese modo, así como la guerra, el hombre es una multiplicidad, un devenir, una cuestión relativa.

\section{b) Convergencias específicas.}

-Si la guerra para Kant, en cuanto es aceptada por un consentimiento general, constituye una decisión en la que deben contemplarse los riesgos, la destrucción y el sufrimiento que conlleva, para el que entra en ese "juego maligno", en el que se puede perder de todos modos $^{3}$, constituye un error. De manera semejante, para Clausewitz, la guerra en tanto está inmersa en el azar, en la buena o mala fortuna, constituye un juego, una apuesta en la que se pone en riesgo la existencia de un pueblo, en la que se juega con el riesgo de ganar o perder. No significa esto que la guerra sea mala, lo que pasa es que así se la juegan los Estados para poder sobrevivir ${ }^{4}$.

\footnotetext{
${ }^{3}$ KANT, I. La Paz Perpetua. Tecnos. Madrid, 1998. Sexta edición. p. 17

${ }^{4}$ CLAUSEWITZ, KV. De la guerra. Idea Books. Barcelona, 1999. Primera edición. Libro 1. Capítulo 1. Num. 21. pp. 44-45.
} 
-Para Kant, si la guerra, como recurso de la naturaleza, va garantizando mayores progresos o avances hacia la paz perpetua, mayor legalidad se puede dar entre los pueblos, mayores interacciones benéficas, porque la guerra es, en cierta forma, un camino al progreso, un factor motivador de mejores condiciones precisamente provenientes de sus efectos negativos 5 .

-De forma análoga, Clausewitz muestra que la guerra, en tanto recurso para realizar los fines políticos, puede garantizar el desarrollo de un pueblo, mientras resulte vencedor y sus fines sean concretados. El progreso es factible en la guerra para el que obtiene las mayores ventajas en la confrontación. Significa que la guerra puede ser la condición de posibilidad para el desarrollo político, moral y cultural de un pueblo; es la fuerza motriz para alcanzar un estatus político en el concurso de las naciones ${ }^{6}$.

-Para Kant es posible ver pueblos, que sin estar organizados bajo el republicanismo, al estar orientados de modo despótico, pueden motivar guerras (por capricho del gobernante), por motivos fútiles. En esa situación la guerra es objeto de glorificación, a pesar de ser un móvil de la maldad humana?

-En términos semejantes Clausewitz muestra que cualquier pretexto, desventaja, deshonor o afrenta pueden causar una guerra de grandes proporciones. Si la guerra se produce por cualquier motivo, es porque la importancia de ellos es relativa, es decir, es otorgada por los intereses fundamentales de una nación o de un gobierno; el motivo fútil se convierte así en fuerza suprema, en asunto de Estado. Todo depende de las circunstancias y de quien esté en contra de dichos propósitos. De esto se concluye que la guerra no es sólo un último recurso, puede ser el primero, de acuerdo con los procesos políticos que estén imperando. El motivo aparentemente vano, puede moldear la forma de hacer la guerra; y si el motivo no es lo suficientemente significativo, la guerra, empero, va a reducir sus manifestaciones de hostilidad. Las guerras van a estar sujetas a gradaciones ${ }^{8}$.

-El Estado como ente circunscrito a la situación natural, y que se otorga su propia ley, sigue teniendo como prerrogativa el entablar una guerra o el optar por la paz. Él mismo no se puede prohibir esta decisión, así como tampoco se puede negar a la opción de la paz; de hecho es un deber acordarla, afirma $\mathrm{Kant}^{9}$, en la caracterización de la teoría política prudente.

-En concordancia análoga, Clausewitz muestra que no existe ni va a existir ningún obstáculo (sea el derecho de gentes o el que sea), que impida o niegue la decisión de la guerra en un Estado. Pero, matizando, Clausewitz muestra que la decisión por las armas es

\footnotetext{
${ }^{5}$ KANT, I. Op. Cit. pp. 33-36

${ }^{6}$ CLAUSEWITZ, K.V. . Op. Cit. Libro 3. Capítulo 3-7

${ }^{7}$ KANT, I. Op. Cit. pp. 36-37

${ }^{8}$ CLAUSEWITZ, K.V. Op. Cit. Libro 3. Cáp. 16. p. 234

${ }^{9}$ KANT, I. Op. Cit. p. 47
} 
signo de la autonomía política de un pueblo, es una necesidad cuando se ve amenazada su existencia. La opción por la guerra, y por la paz en caso de inminente destrucción, siguen siendo una cualidad o disposición consustancial de todos los Estados ${ }^{10}$.

-Por último se halla una convergencia en cierta forma de reprobación respecto al uso de medios crueles que puedan disminuir el fin político y degradar la confrontación, impidiendo la alternativa de la paz, y haciendo disminuir la importancia de las finalidades políticas.

\section{Divergencias y contraposiciones.}

\section{a) Diferencia general.}

El plano de reflexión de Kant es el de la filosofía práctica expresada en la ética (moral) del deber de que no haya guerra, para ello plantea una constitución republicana en el interior del Estado, y en su filosofía de la historia, una confederación de estados que ofrezca garantías de paz. En el derecho propone una clasificación que comprende el derecho interno, el derecho de gentes y el derecho cosmopolita. En la integración de todos estos aspectos se hace visible la fundamentación teórica y la posibilidad de concreción de la paz perpetua.

En contraste, la base para la reflexión de Clausewitz sobre las condiciones esenciales de la guerra, se configura por el ámbito de la teoría política y por la teorización del fenómeno a partir de los datos de la experiencia histórica o de la vivencia directa. Así mismo presenta una visión que brinda elementos que pueden corresponder con una descripción antropológica, a partir de los elementos constitutivos de toda confrontación ${ }^{11}$.

\section{b) Divergencias y contraposiciones específicas.}

La divergencia puede ser fruto de puntos de vista encontrados, pero que pueden contar con ciertos matices; la contraposición o antagonismo podría mostrar puntos de vista, en relación con la problemática de la guerra y la paz, que son diametralmente opuestos y donde, probablemente, no cabrían los matices. En ese sentido, estas podrían ser las diferencias más específicas y relevantes:

\footnotetext{
${ }^{10}$ CLAUSEWITZ, K.V. Op. Cit. Libro 1. Cáp. 1. Num. 2. p. 29

${ }^{11}$ En cuanto análisis con aspiración de universalidad, la teoría tiene como referente la experiencia, lo que podría acercarlo a Kant, en el planteamiento epistemológico relacionado con la forma como se construye el conocimiento, y que se aplica para el análisis del fenómeno de la guerra. Véase a este respecto el artículo: Epistemología de Kant en Clausewitz, por Antonio Cavalla, en la página Web de pensamiento político denominada Catoblepas.
} 
-Aunque parezca obvio, el antagonismo de mayor significado es el que corresponde con la posibilidad de la paz perpetua, que en Kant es un camino, una realización progresiva. En contraposición, Clausewitz muestra que la guerra siempre ha sido y será la constante del devenir político de los pueblos. Esa es la verdad fundamentada en la experiencia histórica. Nunca ha reinado ni reinará la paz en el mundo; la paz es una excepción, una circunstancia efímera, un estado de transición hacia otras guerras.

Para Kant, la paz perpetua no es fruto directo de la fuerza, es resultado de una concertación; el estado de paz es instaurado por el acuerdo general que permite la salida del estado de naturaleza y conduce a los pueblos a ingresar a la confederación que asegure la permanencia de la paz, de una vez por todas. En contraposición, Clausewitz muestra que no hay una sola paz que no haya provenido de alguna guerra. En otras palabras, la paz, vista desde el plano de los tratados, es resultado de la imposición del actor armado que obtuvo mayores ventajas. El acuerdo universal para no recurrir a la fuerza es totalmente descabellado, porque no corresponde con la realidad del fenómeno. Sería, por tanto, una idea falsa e incongruente.

-Mientras Kant indica que la paz es un bien por sí mismo, y la guerra un mal ${ }^{12}$, Clausewitz muestra que así como la guerra es relativa, también lo es la paz; tanto una como otra son cuestiones sujetas a lo probable, al influjo del azar. No puede haber entonces una paz buena en todos los casos; habrá algunas, pero hay las que son prolongadas, que pueden ir en contravía del desarrollo político y moral de una nación ${ }^{13}$.

-Otra discrepancia consiste en que, mientras para Kant la paz es un fin último, un fin absoluto prescrito por la razón moral ${ }^{14}$ y la guerra un medio para garantizar la consecución de ese fin, para Clausewitz, en general, los tratados, que son la única forma en que se expresa la paz, son una continuación del conflicto por la vía de la diplomacia; la paz es una continuación de la tensión de fuerzas, de los cálculos políticos que pueden hacer, entre otras cosas, que no haya una imposición agresiva, o que puede servir como último recurso ante la inminente desaparición, como también para obtener alguna concesión, así se haya perdido la guerra. La paz, en Clausewitz, puede ser a la vez un medio y un fin, pero no un fin absoluto, porque en la guerra y en la vida en general, no existen absolutos ni se opera a partir de ellos ${ }^{15}$.

-En las condiciones preliminares, Kant $^{16}$ plantea que no debe considerarse válido un tratado que contenga la intención oculta que cause otras guerras en el futuro. Clausewitz ${ }^{17}$ muestra que esa es la esencia invariable de los tratados, el no ser salidas definitivas, el estar sujetas a engaños; y si lo fueran aún no eliminarían la posibilidad de guerra; si hubieran tratados

\footnotetext{
${ }^{12}$ KANT, I. Op. Cit. pp. 37,69

${ }^{13}$ CLAUSEWITZ, K. V. Op. Cit. Libro 3. Cáp. 4-5

${ }^{14}$ KANT, I. Op. Cit. pp. 61-69, y suplemento primero.

${ }^{15}$ CLAUSEWITZ, K.V. Op. Cit. Libro 1. Cáp. 1. Num. 9 y 13, y libro 6. Cáp. 26

${ }^{16}$ KANT, I. Op. Cit. p. 5

${ }^{17}$ CLAUSEWITZ, K. V. Op. Cit. Libro 1. Cáp. 1
} 
definitivos, sería porque los estados se encuentran en absoluta quietud política; tendría que admitirse una inmovilidad absoluta de las interacciones entre los pueblos, lo cual nunca ha sido ni podrá ser.

$\mathrm{Kant}^{18}$ establece que los ejércitos permanentes deben desaparecer gradualmente. En contraste, Clausewitz ${ }^{19}$ propone que, en tanto la guerra es connatural y común a todos los pueblos, las fuerzas militares son imprescindibles para garantizar la existencia de un pueblo; y si aún no existieran o fueran aniquiladas el pueblo tendría la necesidad de asumir esta función.

-Kant afirma que la decisión de hacer guerra es un asunto sencillo para el que gobierna de forma despótica ${ }^{20}$. Entre tanto para Clausewitz, lo único fácil en la guerra son sus conceptos generales. Como actividad constituyen una de las acciones más complejas para el entendimiento humano, porque congrega tantos elementos y circunstancias diversas, que cada paso puede convertirse en un asunto de suma gravedad ${ }^{21}$.

-En las relaciones entre guerra y paz, existe otro antagonismo: para Kant una de las garantías de la paz perpetua es el incremento de las relaciones comerciales entre los Estados, que hace que la guerra vaya siendo desechada ${ }^{22}$. Para Clausewitz no hay actividad que tenga mayor parecido y conexión con la guerra que el comercio, pues dicha actividad es, en el fondo, un conflicto de intereses, así como la guerra es una tensión de fuerzas contradictorias y planes políticos que se resuelve por derramamiento de sangre ${ }^{23}$.

Kant propone que uno de los medios o garantías para que la guerra se convierta en algo inviable, es el desarrollo de la cultura, que elimina inclinaciones a la barbarie. El aumento de la cultura es un camino hacia la realización de los fines conducentes a la paz perpetua, un obstáculo a los intentos de agresión ${ }^{24}$. En contraste, Clausewitz muestra que incluso los pueblos más civilizados pueden inflamarse de un odio irracional, que los lleve a cometer acciones de la mayor crueldad contra sus adversarios ${ }^{25}$. Así mismo, el desarrollo de la cultura ha sido un aliciente para mejorar las técnicas de destrucción, a través de los desarrollos de las ciencias. Así, cuanto mayor sea el nivel cultural y político, mayores serán los niveles de hostilidad y destrucción por las guerras que entablen los pueblos civilizados.

\footnotetext{
${ }^{18}$ KANT, I. Op. Cit. pp. 7-8

${ }^{19}$ CLAUSEWITZ, K. V. Op. Cit. Libro 2. Cáp. 2-6

${ }^{20}$ KANT, I. Op. Cit. p. 17

${ }^{21}$ CLAUSEWITZ, K. V. Op. Cit. Libro 2. Pág. 130-131; Libro 1. Cáp. 3

${ }^{22}$ KANT, I. Op. Cit. p. 41

${ }^{23}$ CLAUSEWITZ, K. V. Op. Cit. Libro 2. Cáp. 3. Num. 3

${ }^{24}$ KANT, I. Op. Cit. pp. 53, 59-60

25 CLAUSEWITZ, K.V. Op. Cit. Libro 1. Cáp. 1. Un ejemplo que puede ilustrar esta idea, mas no demostrarla, fue la Alemania de la Segunda Guerra Mundial. Una nación con un elevado desarrollo cultural y científico, fue el centro de la mayor barbarie cometida hasta ahora. Los avances de la ciencia (la medicina, la química, etc.), fueron un instrumento eficaz de las matanzas sistemáticas en los campos de concentración
} 
A juicio de Kant, los medios o acciones excesivas deben prohibirse estrictamente: son reprobables por su incitación a la deshonra y por minar la confianza necesaria para entablar la paz. Por su parte, Clausewitz muestra que, pese a la tendencia de regulación del uso de la fuerza, desde la visión absoluta, donde se justifica la guerra total, el desarrollo del proceso conflictivo puede conducir a los adversarios a límites insospechados de interacción violenta. $^{26}$

Por último, la guerra para Kant no tiene nada de moral (es, de hecho, si se puede decir, antimoral); es contraria a los lineamientos de la razón en todo sentido. Al contrario, Clausewitz muestra que la moral sí tiene cabida en la guerra, cuando se habla de la virtud militar, por ejemplo, donde imperan valores como el orden, la valentía, la audacia, la perseverancia, el cumplimiento del deber, la obediencia, etc. En la guerra es perfectamente aceptable hablar de virtud, es decir, de buenas acciones ${ }^{27}$.

\section{Los conceptos de guerra y paz}

Por lo visto, parece evidente que desde la propuesta de Clausewitz el término guerra es el concepto central, al que está subsumida la noción de paz, haciendo de ésta un término negativo, y por ello, complementario, pero secundario. Negativo porque paz equivaldría a ausencia de guerra. De este modo guerra y paz, en tanto conceptos complementarios conforman una síntesis, donde el elemento fundamental es el del conflicto, en sus manifestaciones de violencia organizada y constante a lo largo de la historia ${ }^{28}$.

En contraste, para Kant, bajo el marco de la obra La Paz Perpetua, la paz viene a ser el término fuerte y la guerra el término negativo, lo cual configura una relación de oposición entre estos conceptos, por medio de la vinculación de guerra con el término caos o maldad, y de paz con el término orden (orden mundial, bien universal); la diferencia parece insalvable. La idea de paz en filosofía, vista desde la justicia, es paz positiva, o lo que es lo mismo, paz verdadera, es decir, permanente en el tiempo y común a toda la humanidad. Aquí la paz no es ausencia de guerra, es una idea independiente, que encarna la visión de un esquema de organización mundial, un orden mundial de cooperación entre los pueblos, que permita zanjar los conflictos sin el recurso de la fuerza ${ }^{29}$.

Entre tanto, para Clausewitz guerra y paz son hechos probables. Así, no es posible identificar la guerra como un mal, y la paz como un bien, porque en la experiencia pueden suceder cosas que muestren lo contrario. Incluso, en sí mismos guerra y paz no son buenos

\footnotetext{
${ }^{26}$ Ibíd.

${ }^{27}$ Ibid. Libro 3. Cáp. 3-5

${ }^{28}$ Para este tipo de acercamiento se ha tomado como base de interpretación el artículo de Norberto Bobbio titulado "Paz". En: Diccionario de Política. Siglo XXI. Tomo 2. México, 1986. pp. 1195-1203

${ }^{29}$ Véase el artículo de Johan Galtung titulado "Paz". En: Enciclopedia Internacional de las Ciencias Sociales. Obra dirigida por David Sills. Aguilar. Tomo 7. Madrid. pp. 686-693
} 
ni malos, son simplemente realidades probables que dependen de las interacciones históricas imperantes y de los resultados favorables o desfavorables, efecto de la confrontación y de las condiciones de los tratados de paz. La guerra como hecho común y la paz como objetivación de un tratado, que puede dar lugar a encender las causas de otras guerras, en eventos posteriores. Así mismo, no constituyen valores más que en los casos específicos, es decir, más que en las interacciones particulares de los adversarios. Así, no se puede decir que la paz sea un valor y la guerra un antivalor; todo depende de la valoración que hagan de sí mismos, y de sus contradictores, los actores armados, los pueblos inmersos en la confrontación ${ }^{30}$; todo depende de la dialéctica de contrarios y la conclusión del antagonismo.

$\mathrm{Al}$ contrario, la paz perpetua es para Kant un bien absoluto, un valor supremo, en tanto que su realización paulatina es el signo de la realización integral de la especie, de la concreción respecto a la tendencia o inclinación histórica del progreso. La guerra en tanto es absolutamente injustificable por la razón moral, representa, por así decir, la encarnación de toda la perversión humana; no puede ser un valor, sino un signo o una fuerza de la degradación ${ }^{31}$. Y aunque en las vías que conducen al progreso, a la integración definitiva de los pueblos, la guerra es un instrumento de la naturaleza, por sí mismo no es un avance, es un retroceso, pero no deja de ser un recurso para llegar a la finalidad suprema de la paz perpetua $^{32}$. A la inversa, en relación con los medios y los fines, la paz y la guerra para el pensador militar, pueden ser a la vez medio y fin de la interacción humana. La paz puede ser el fin último de la estrategia, y a la vez, en tanto se presente el tratado de paz, éste puede ser un medio para obtener ventajas o para salvaguardar lo que le queda a un Estado después de una guerra con desventaja. Entre tanto, la guerra es un medio de la política, un instrumento que tiene sus finalidades intrínsecas, aunque no pierde su carácter. Por esencia, la guerra es un medio aunque contenga finalidades intrínsecas, que la convierten, en cierta manera, en un fin.

En suma, en Clausewitz guerra y paz son conceptos que se entrecruzan, que aluden a una misma realidad, a un mismo devenir: el de las interacciones políticas, que constituyen un fenómeno polimorfo; en tanto conforman el sustrato de la vida social, guerra y paz son casi una misma cosa. En cambio, para Kant, la paz es un deber diferenciado, una aspiración (la más fundamental), de las relaciones entre los pueblos. La paz es un concepto diferenciado, con su propia esencia filosófica (ético-político), y por ello una exigencia para la especie humana; un mandato proferido por la razón moral que no permite negaciones. La guerra es el hecho, la constante que se le opone en cuanto concepto y aspiración. La guerra se inscribe y es proyección de la naturaleza perversa del hombre; la paz es la exigencia de la razón que hace al hombre verdaderamente libre y autónomo. Guerra y paz son, hasta algún punto, ideas irreconciliables.

\section{Conclusión}

\footnotetext{
${ }^{30}$ BOBBIO, N. Op. Cit. pp. 1199-1200

${ }^{31}$ Ibíd.

${ }^{32}$ Ibíd. pp. 1200-1201
} 
Pese a los puntos en común en los planteamientos respecto al reconocimiento de la guerra como constante, si se puede decir, en lo que corresponde con la guerra, que los puntos de vista de Kant y Clausewitz son irreconciliables en lo que toca a la posibilidad de una paz universal, de una congregación armónica de todos los pueblos de la tierra. No obstante, lo que es cierto es la interdependencia de los dos términos en el esclarecimiento de sus límites conceptuales, en su vínculo a lo que se podría llamar la condición trágica de la realidad humana, la necesidad de la lucha, la inclinación natural combativa, a la vez que el temor a tener que llevarla a cabo.

Esto sugiere que la guerra no ha dejado ni dejará de hacer parte, como afirma Clausewitz, de la cadena de las debilidades humanas, mientras que la paz no dejará de hacer parte de las esperanzas o aspiraciones en momentos comprensión del sin sentido de una situación de conflicto armado. Así se hacen necesarias las prospecciones de un mundo en otras condiciones, se hace imprescindible la utopía de la paz duradera, aunque esto ignore, en alguna forma, el carácter trágico y contradictorio de las interacciones humanas.

\section{BIBLIOGRAFÍA}

ARON, Raymond. Paz y guerra entre las naciones. Revista de occidente. Madrid, 1975 BEHAR, Olga. Las guerras de la paz. Círculo de lectores. Bogotá, 1985. BOBBIO, Norberto. Diccionario de política. Siglo XXI. Dos tomos. México, 1986. BORJA, Rodrigo. Enciclopedia de la política. F.C.E. México, 2002. Dos Tomos. BOUTHOUL, Gastón. La guerra. Oikos-Tau. Barcelona, 1983.

CANTO-SPERBER, Monique. Diccionario de ética y filosofía moral. Fondo de Cultura Económica. Dos tomos. México, 2004

CLAUSEWITZ, KV. De la guerra. Idea Books. España, 1999.

DEPARTAMENTO DE SOCIOLOGÍA. Revista Colombiana de Sociología. Universidad Nacional de Colombia. Volumen 5. Número 7. Bogotá. 2000.

EQUIPO DE REDACCIÓN PAL. Historia universal. Bolsillo mensajero. Tomo 7. Bilbao, 1985.

GIRALDO IZAZA, Fabio. (Compilador). Guerra y muerte. Iepri - Fica. Bogotá, 2002.

HERRERA, Wilson, de GAMBOA, Camila (Compiladores). Kant: Defensa y límites de la razón. Universidad del Rosario. Escuela de Ciencias Humanas. Bogotá. 2005

IEPRI-FICA. Guerra en Colombia-actores armados-. Bogotá, 1998

KAGAN, Paul. Sobre las causas de la guerra y la preservación de la paz. Fondo de Cultura Económica. España, 2005.

KANT, I. Sobre la paz perpetua. Traducción de Antonio Truyol y Serra. Tecnos. Madrid, 1998.

--------- Principios metafísicos del derecho. América Lee. Obra a cargo de Francisco Ayala. Buenos Aires, 1943.

MONTGOMERY, Sir Bernard Law. Historia del arte de la guerra. Aguilar. Madrid. 1969. POSADA CARBÓ, Eduardo. ¿Guerra civil en Colombia? El lenguaje del conflicto armado. Alfa Omega. Bogotá, 1997. 
STERLING, Mc. Murrin (Compilador). Valores en guerra - un debate sobre la crisis nuclear. F. C. E. México, 1987.

STRAUSS, Leo; CROPSEY, Joseph (Compiladores). Historia de la filosofía política. F.C.E. México, 1993

SUN TZU. El arte de la guerra. Edición crítica de Samuel Griffith. Evergreen. Oxford, 2005.

TREUE, W.. Historia de Alemania. UTEHA. Tomo 7. México, 1964.

VALLESPÍN, Fernando (Editor). Historia de la teoría política. Alianza. Madrid, 2002. Tomo Tres.

VILLAR BORDA, Luis. La paz en la doctrina del derecho de Kant. Universidad Externado de Colombia. Bogotá, 1998. 